\title{
Examining the M67 Classification as an Open Cluster
}

\author{
Shim'on Naim ${ }^{1}$, Evgeny Griv ${ }^{2}$ \\ ${ }^{1}$ Ilan Ramon Physics Center, Ben-Gurion University of the Negev, Beer-Sheva, Israel \\ ${ }^{2}$ Department of Physics, Ben-Gurion University of the Negev, Beer-Sheva, Israel \\ Email: shimonnaim@yahoo.com,griv@bgu.ac.il
}

Received May 22, 2012; revised June 28, 2012; accepted July 15, 2012

\begin{abstract}
The cluster M67 (= NGC 2682) in Cancer is a rich stellar cluster, usually classified as an open cluster. Using our own observations with the $0.4 \mathrm{~m}$ telescope, we show that M67 is a tight group of about 1200 stars. The actual radius of the cluster is about $3.1 \mathrm{pc}$ and the average mass of a star in the system is about $1.33 \mathrm{M}_{\odot}$. We also show that the ratio of the mean kinetic energy of the cluster to its mean gravitational potential energy $K_{\text {clust }} / U_{\text {clust }} \approx-0.3$, while the value predicted by the virial theorem is equal to -0.5 . So the system is a gravitationally bound. This value of $K_{\text {clust }} / U_{\text {clust }}$ is considered as an evidence of quasi-stability of the cluster and allows us to use the Chandrasekhar-Spitzer relaxation time for M67 $\tau_{\text {chand }} \approx 30$ Myr as a characteristic dynamical relaxation time of the system under study. As the cluster is almost twice older its half-life time $t_{\text {half }} \approx 70 \tau_{\text {chand }}$, it is argued that M67 was in the past (about 4 Gyr ago, close to its formation) a relatively small ( $\sim 10,000$ stars) globular cluster, but got "open cluster" shape due to the dynamical evaporation of the majority of its stars.
\end{abstract}

Keywords: Photometers; Techniques; Photometric; Open Clusters and Associations; M67; Galaxies; Star Clusters

\section{Introduction}

Usually classified as an open cluster, the dense stellar cluster M67 in Cancer is one of the oldest known open clusters in our Galaxy with an age $t_{\text {clust }}$ of 3.5-4.5 Gyr, that is, close to the age of the sun [1-3]. In the present paper we adopt the value $t_{\text {clust }}=4$ Gyr $[4,5]$. It is important to note that open clusters usually get destructed much faster, over $\sim 10^{8} \mathrm{yr}$ timescales. It is some $750-850$ pc distant the Sun. Following Ref. [6], we adopt the distance $r_{\text {clust }}=770$ pc. The total number of stars in M67 is estimated at over 700 [7]. M67 is the nearest old open cluster. The cluster is also anomalous with respect to the distance of $z_{\text {clust }} \approx 600 \mathrm{pc}$ to the Galactic equatorial $z=0$ plane, where most open clusters are found. This distance $z_{\text {clust }}$ is even larger than scale height $h=300-400 \mathrm{pc}$ of the local thick disk of the Galaxy (see, e.g. Ref. [8]). Other open clusters lie much closer to the Galactic plane. M67 current mass is $M_{\text {clust }} \approx 1400 M_{\odot}$ and its initial mass was to be approximately $M_{\text {past }}=10 M_{\text {clust }}$ [9]. Mass segregation has been suggested as the cause for the observed structural properties of M67 in Refs. [10,11]. This process has long been suspected for globular star clusters (and has been directly seen in globular cluster 47 Tucanae [12]), but has never been suggested for open clusters. We conclude that M67 has properties of globular clusters.
Here, we study M67 with the use of the Meade LX200R robotic $0.4 \mathrm{~m}$ telescope of the Ilan Ramon Physics Center Observatory in Israel (http://ilanramon.bgu.ac.il) (Figure 1). The telescope operates from a small dome and is equipped with Meade DSI III pro $1360 \times 1024$ CCD camera (Figure 2). In the last four years this telescope was intensively used in a variety of monitoring projects, including the study of stellar clusters, in particular, M67 and M35 (=NGC 2168).

\section{Observations}

The cluster M67 was photographed in two filters, $V$ and $B$, on 7th April 2010. An (f/6.3) local reducer was used, and therefore the scale is $0.52 \mathrm{arcsec} / \mathrm{pixel}$. Two sets of exposure times were used in the observations, namely, long (10 s in both $B$ and $V$ filters) and short ( $3 \mathrm{~s}$ in both $B$ and $V$ filters).

Figure 3 shows a part of M67. This photograph is a combination of 13 frames from each exposure, $10 \mathrm{~s}$ and 3 $\mathrm{s}$, summed after a registration. The picture was taken with a blue Johnson filter. First, we estimated the angular size of the cluster $\Delta \varphi=27.6 \pm 3.6$ arcsec. Then, adopting the revised distance to the cluster $r_{\text {clust }}=770 \mathrm{pc}$, its actual radius was found $R_{\text {clust }}=3.1 \pm 0.26 \mathrm{pc}$. In order to cast more light on the structure of M67, in Figure 4 we show a wider field $8^{\prime} \times 16^{\prime}$ photograph of M67, 


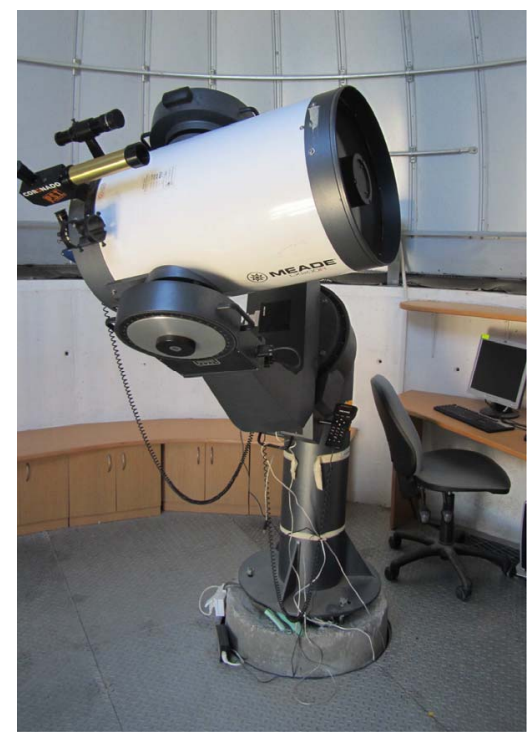

Figure 1. The Meade LX200R robotic 16" telescope of the Ilan Ramon Physics Center Observatory in Beer-Sheva (Israel).

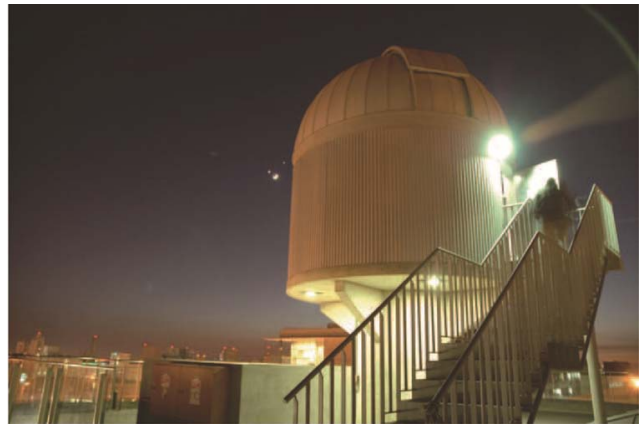

Figure 2. The dome of the Meade LX200R robotic 16" telescope.

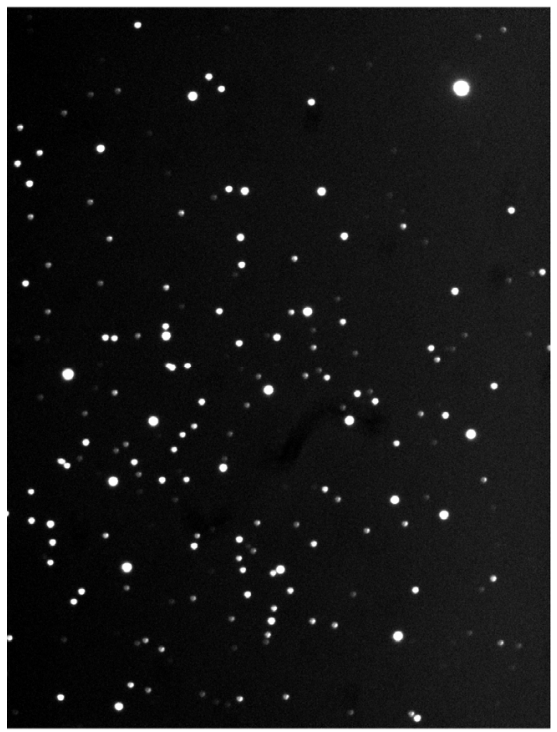

Figure 3. This photograph shows 173 stars of M67 cluster in our observations.

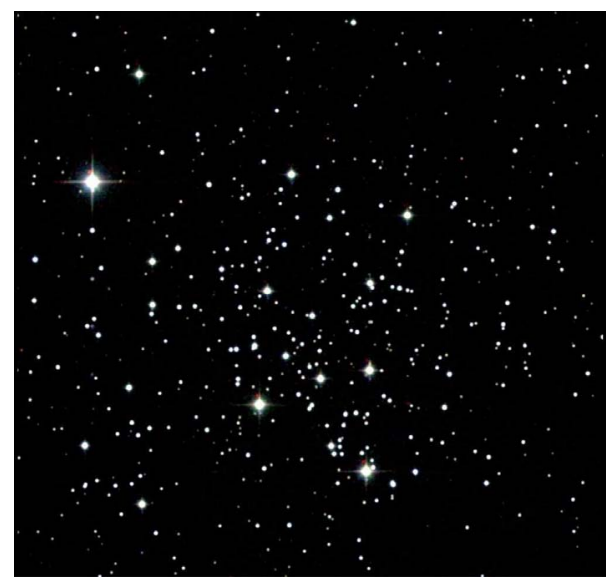

Figure 4. A wider field $8^{\prime} \times 16^{\prime}$ photograph of M67, taken from the Atlas Image website.

taken from the Atlas Image website. Using these photographs (Figures 3 and 4 ) and this $R_{\text {clust }}$, finally we estimated the total number of stars in the system

$N_{\text {clust }} \approx 1200$.

Figure 5 shows the observed color-magnitude, $m_{\text {vis }}$ vs. $(B-V)$, diagram (or the HR diagram) for M67, based on our observations. Each dot denotes a star. The purple color indicates the positions of the main sequence stars. The red color indicates the positions of the horizontal branch stars. The yellow color indicates the positions of the giant stars. The positions of blue stragglers are indicated in the diagram by the blue color. As is seen, the color-magnitude diagram for M67 shows a short main sequence extending from $(B-V) \approx 0.4$, where the turn-off point is located, up to $(B-V) \approx 0.7$. After $(B-V) \approx 0.7$ it starts merging in the field region stars and is not seen clearly. The giant branch is also clearly seen. Figure 5 evidently illustrates that M67 is quite old cluster. There are stars that are brighter and bluer than the main sequence stars and giant branch stars. These stars are a part of an exotic class of blue stragglers. A spectroscopic study of the blue stragglers in M67 has been done in Ref. [13]. Notice that the HR diagram shown in Figure 5 is more typical for globular clusters than for open clusters.

Figure 6 presents our photometric color-magnitude diagram for the "ordinary" open cluster M35. (The cluster age is about $130 \mathrm{Myr}$ [14].) In sharp contrast to M67, the color-magnitude diagram for M35 clearly shows a well-defined long main sequence stretching from $(B-V) \approx-0.1$ to $(B-V) \approx 1.4$, but does not show horizontal branch stars, the giant branch stars, and blue stragglers. Such an HR diagram is typical for open clusters.

Thus, the following parameters were derived from our observations: $\Delta \varphi=27.6 \pm 3.6$ arcsec, $R_{\text {clust }}=3.1 \pm 0.26$ pc (adopting $r_{\text {clust }}=770 \mathrm{pc}$ ), and $N_{\text {clust }} \approx 1200$. 


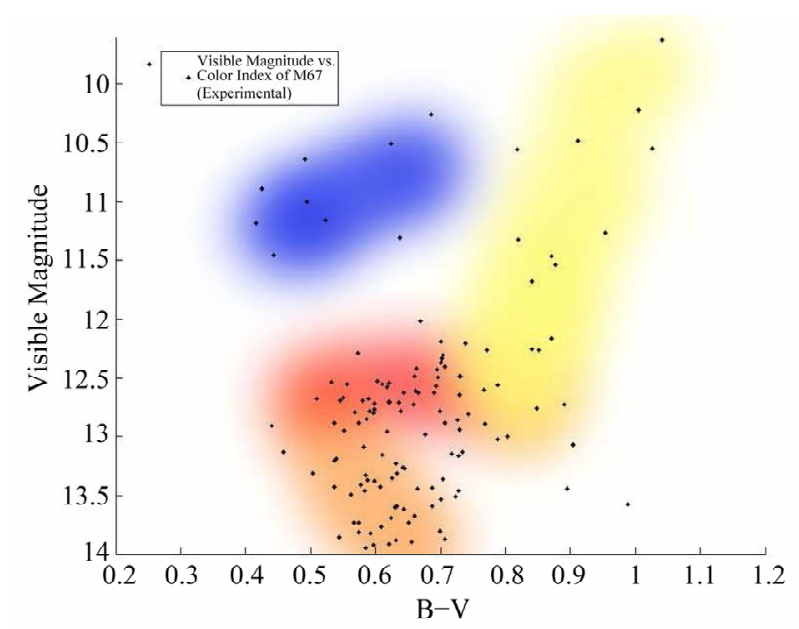

Figure 5. The color-magnitude diagram for M67, showing the apparent magnitude $m_{\text {vis }}$ of stars versus their color in$\operatorname{dex}(B-V)$.

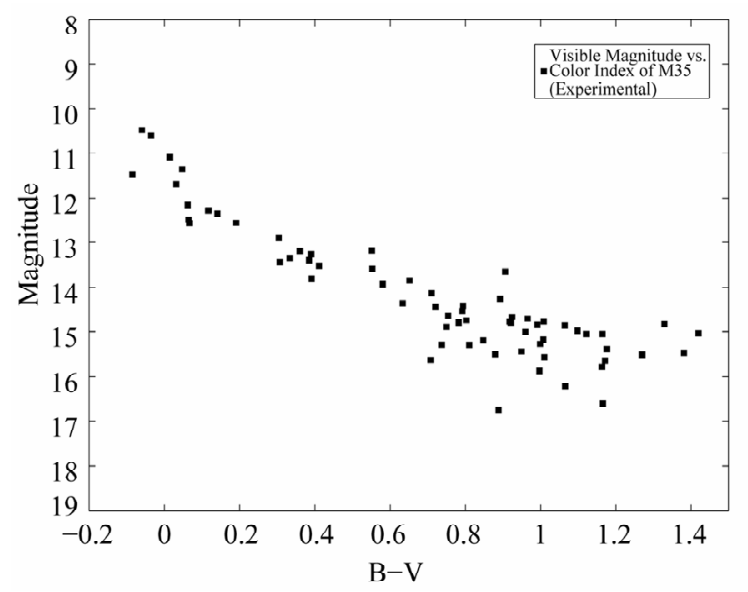

Figure 6. The color-magnitude diagram for M35 (cf. Ref. [14]).

\section{Total Energy of M67}

One of the principal features of stellar clusters is their quasi-stability: Hundreds of millions of years may elapse before the cluster will begin to disintegrate by some stars acquiring velocities exceeding the escape velocity $v_{\text {esc }}$. This is because the total energy of a cluster in a quasisteady state is negative. Let us show that the assumption of a quasi-steady state for M67 is reasonable.

The average mass of a main-sequence star in the cluster $M_{\text {star }}$ was evaluated by using the standard massluminosity relation $L \propto M^{3.5}$. From the definition $m_{\text {abs }}=-2.5 \log L+$ const, one gets the following massabsolute-magnitude $\left(M-m_{\text {abs }}\right)$ relation:

$$
M\left(m_{\text {abs }}\right)=M_{\odot} \times 10^{\left(m_{\text {abs }, \odot}-m_{\text {abs }}\right) / 8.75} .
$$

From the HR diagram (Figure 5) and Equation (1), one can estimate the average mass of a star in the cluster

$$
M_{\text {star }}=\frac{1}{N_{\text {clust }}} \sum_{i=1}^{N_{\text {clust }}} N_{i} M\left(m_{\text {abs }, i}\right) .
$$

As is seen, however, this diagram is too noisy for the dimmest stars (and some of the dimmest stars are not shown in Figure 5 due to a high noise). Therefore, we prefer to use a much more simple model. Namely, examining our and other (e.g. Ref. [1]) HR diagrams, one can see that the main sequence of this cluster is occupied mainly by stars with absolute magnitude ranging from $m_{\mathrm{abs}}=3$ to $m_{\mathrm{abs}}=6$, with more stars closer to $m_{\mathrm{abs}}=3$. Also, from Figure 5 we obtained that the number of stars $N\left(m_{\mathrm{abs}}=3\right) \approx 2 N\left(m_{\mathrm{abs}}=6\right)$. Therefore, in the lowest approximation the cluster consists only of stars with absolute magnitude $m_{\text {abs }}=3$. In the next approximation, the average mass of a star is obviously

$$
M_{\mathrm{star}} \approx \frac{2 M\left(m_{\mathrm{abs}}=3\right)+M\left(m_{\mathrm{abs}}=6\right)}{3} .
$$

Taking $m_{\text {abs }, \odot} \approx 4.85$, we get $M_{\text {star }} \approx 1.33 M_{\odot}$. Finally, using $M_{\odot}=1.989 \times 10^{33} \mathrm{~g}$, we have

$$
M_{\text {star }} \approx(2.65 \pm 0.9) \times 10^{33} \mathrm{~g} .
$$

This average mass of a star is in good agreement with most recent studies of M67.

In order to estimate the kinetic energy, an average speed of a star was calculated as

$$
v_{\text {star }}=\sqrt{v_{r}^{2}+v_{\varphi}^{2}} .
$$

The typical radial velocity is $v_{r}=(0.48 \pm 0.15) \times 10^{3}$ $\mathrm{m} \cdot \mathrm{s}^{-1}$ and the transverse velocity is $v_{\varphi}=(0.81 \pm 0.1) \times 10^{3} \mathrm{~m} \cdot \mathrm{s}^{-1}$ [7]. This value of $v_{\varphi}$ is based on the distance $r_{\text {clust }}=870 \mathrm{pc}$ [7]. We corrected $v_{\varphi}$ to a new one based on the distance $r_{\text {clust }}=770 \pm 100$ pc by taking $v_{\varphi}=(0.81 \times 770 / 870) \mathrm{km} \cdot \mathrm{s}^{-1}$. From Equation (4) one easily obtains

$$
v_{\text {star }}=(0.86 \pm 0.2) \times 10^{3} \mathrm{~m} \cdot \mathrm{s}^{-1} .
$$

The kinetic energy of the cluster $K_{\text {clust }}$ would then be

$$
K_{\text {clust }}=N_{\text {clust }} \frac{M_{\text {star }} v_{\text {star }}^{2}}{2}=(1.1 \pm 0.6) \times 10^{39} \mathrm{~J} \text {. }
$$

The average gravitational potential energy $U_{\text {clust }}$ was calculated using the well-known relation for energy of a self-attracting spherically symmetric mass $M$. The gravitational potential energy of the mass $M$ of the radius $R$ is given by

$$
U \approx-\frac{3}{5} \frac{G M^{2}}{R} .
$$

For the cluster we use $M_{\text {clust }}=N_{\text {clust }} M_{\text {star }}, R=R_{\text {clust }}$ and get $U_{\text {clust }}=-(3 / 5)\left(G M^{2} N_{\text {clust }}^{2} / R_{\text {clust }}\right)$. This immediately implies that the potential energy of the cluster is 


$$
U_{\text {clust }}=-(4.3 \pm 3) \times 10^{39} \mathrm{~J} .
$$

Thus, the total energy of M67,

$$
E_{\text {clust }}=K_{\text {clust }}+U_{\text {clust }} \approx-3.2 \times 10^{39} \mathrm{~J} \text {, }
$$

is negative but small in its absolute value in a sense that the kinetic energy and the potential energy are of the same order of magnitude. Moreover, the value of $K_{\text {clust }} / U_{\text {clust }} \approx-0.3$, while the value predicted by the virial theorem for a stable system consisting of $N$ particles, bound by potential forces, is $K_{\text {clust }} / U_{\text {clust }}=-0.5$. From the above, one concludes that the assumption of a quasi-steady state for M67 is indeed reasonable. This is an important fact from the point of view of stellar dynamics. One understands, however, that even though the cluster is dynamically bound, $E_{\text {clust }}<0$, escaping of stars from the system due to the Maxwellian-like velocity spread is possible (see Section 4 below for an explanation).

\section{Evaporation of Stars from M67}

Many factors cause stars to dissipate from dynamically bound clusters. In Ref. [15], it has pointed out that because of the scattering of stars in clusters on the stars in the galactic field the total internal energy of a cluster should rise, ultimately leading to a complete breakup of the cluster. It turned out that the characteristic time for this process of destruction of dense stellar clusters is at most $10^{10}$ yr. Spitzer $[16,17]$ has calculated the increase of energy leading to desintegration of a cluster due to encounters with interstellar clouds. Spitzer has argued that the characteristic time for a process of this sort is also $10^{10} \mathrm{yr}$. The importance of the galactic tidal force on the evolution and stability of clusters has been also discussed (e.g. Ref. [18]). The characteristic decay time for this process is more than $10^{9} \mathrm{yr}$. We conclude that the time $t_{\text {age }}=10^{10} \mathrm{yr}$ might be regarded as an upper limit on the age of clusters in the Galaxy.

Other investigations have studied the most important mechanism for the destruction of dense stellar clusters, namely, the dissipation which is caused by the interaction of stars as they approach one another [19-22]. See also Refs. [23-25] for a discussion of the problem. Evidently, in this process energy exchange among cluster members will cause individual stars to acquire supercritical velocities (escape velocities) of the order of

$v_{\text {esc }} \approx 2 \sqrt{\left\langle v^{2}\right\rangle}$, where $\sqrt{\left\langle v^{2}\right\rangle}$ is the rms velocity) in excess of the parabolic velocity and actually to leave the cluster, whose total mass therefore decreases. The escaping stars carry away a positive energy and a state of statistical equilibrium is impossible for a cluster containing a finite number of stars if the interactions between stars is strictly taken into account [26,27]. See also Ref.
[28] for a discussion.

By the time of relaxation of star systems, generally speaking we mean the characteristic time of approach of the distribution function $f(\boldsymbol{r}, \boldsymbol{v}, t)$ of the stars with respect to peculiar (random) velocities $\boldsymbol{v}$ to a Maxwellian distribution [22]. (The distribution function $f(\boldsymbol{r}, \boldsymbol{v}, t)$ is defined by the condition that $f(\boldsymbol{r}, \boldsymbol{v}, t) \mathrm{d} \boldsymbol{v} \mathrm{d} \boldsymbol{r}$ is the number of stars at time $t$ in volume element $\boldsymbol{r}, \boldsymbol{r}+d \boldsymbol{r}$ and in velocity element $v, v+d v$.) The time of establishment of a Maxwellian distribution of random velocities as a result of encounters between test stars $M_{\text {test }}$ and field stars $M_{\text {field }}$ (the "collisional" relaxation time) has the order of magnitude [22,23]

$$
\tau_{\text {chand }}=\frac{v^{3}}{10 G^{2} M_{\text {field }}^{2} n \log \Lambda},
$$

where $v$ is the relative velocity, which is approximately the velocity dispersion of the lighter stars, $n$ is the number density of the field stars, $M_{\text {test }}$ and $M_{\text {field }}$ are the masses of test and field stars, $\log \Lambda$ is the so-called Coulomb (Newton) logarithm, by means of which the longrange nature of the gravitational force is taken into account, $\Lambda \approx 0.4 N_{\text {clust }}$, and $N_{\text {field }}$ is the total number of field stars in the system. If we assume that the stellar distribution in the system tends to a Maxwell-Boltzmann distribution as a result of stellar encounters, during relaxation time the system loses approximately 0.0074 of its members (e.g. Ref. [28]). Thus we can properly regard $t_{\text {half }} \approx 70 \tau_{\text {chand }}$ as a measure of the half-life time of a cluster. (Although the general conclusions on the relaxation time reached by Chandrasekhar, Spitzer, and others are correct, the discussions were based on a simplified "molecular-kinetic" theory and not on an explicit solution of the time-dependent problem. In Appendix 8, we follow numerically the relaxation of an isotropic system of like stars through small-angle Coulomb encounters by using the more accurate approach.)

In M67, assuming $M_{\text {field }} \approx M_{\text {test }} \approx M_{\text {star }}=1.33 M_{\odot}$ (Equation (3)), $v \approx v_{\text {star }}=0.86 \mathrm{~km} \cdot \mathrm{s}^{-1}$ (Equation (5)), and $n=3 N_{\text {clust }} / 4 \pi R_{\text {clust }}^{3}$, one obtains $\tau_{\text {chand }} \approx 3 \times 10^{7}$ yr for star-star gravitational encounters, So that the half-life time of the cluster is relatively short, of the order of $t_{\text {half }}=2 \times 10^{9} \mathrm{yr}$ only. (We estimate that the dynamical Chandrasekhar-Spitzer relaxation time for M67 is $3 \times 10^{7} \mathrm{yr}$, which implies that the cluster age is 133 times its relaxation time. In contrast for the "ordinary" open cluster M35, the cluster age $t \approx 1.3 \times 10^{8} \mathrm{yr}$ is only 1.6 times its relaxation time [14].) We argue therefore that in the past $\left(\sim 4 \times 10^{9} \mathrm{yr}\right.$ ago $)$ the cluster M67 was a relatively small $(\sim 10,000$ stars $)$ globular cluster, but got "open cluster" shape due to the dynamical evaporation of a substantial portion or even the majority of its stars. Interestingly, the direct $\mathrm{N}$-body model with 36,000 stars for M67, evolved from zero age to $4 \mathrm{Gyr}$, 
has been presented in Ref. [9]. It has been shown that at 4 Gyr the total mass has reduced to $2000 M_{\odot}$ as a result of mass loss and stellar escapes.

\section{Concluding Remark}

Summarizing, from our own observations, we built the HR diagram for the cluster M67. We showed that this diagram is more typical for relatively old globular clusters than for open clusters. We also estimated the total number of stars in the cluster $N_{\text {clust }} \approx 1200$, its actual radius $R_{\text {clust }} \approx 3.1 \mathrm{pc}$, and the average mass of a star $M_{\text {star }} \approx 1.33 M_{\odot}$. We studied the dynamical features of M67 and argued that

- The total energy of the cluster $E_{\text {clust }}=K_{\text {clust }}+U_{\text {clust }}$ is negative, $E_{\text {clust }} \approx-3.2 \times 10^{39} \mathrm{~J}$. Moreover $K_{\text {clust }} / U_{\text {clust }} \approx-0.3$. The latter may be considered as evidence of the quasi-stability of the system under consideration.

- The cluster is a tight group of about 1200 stars which are gravitationally bound, $E_{\text {clust }}<0$.

- As the cluster is quasi-stable, its characteristic relaxation time is the Chandrasekhar-Spitzer time $\tau_{\text {chand }} \approx 3 \times 10^{7}$ yr and the half-life time is equal to $t_{\text {half }} \approx 70 \tau_{\text {chand }} \approx 2 \times 10^{9} \mathrm{yr}$, respectively.

- As the cluster is almost twice older its half-life time, we conclude that many stars have left the cluster since its formation. In the past $\left(\sim 4 \times 10^{9} \mathrm{yr}\right.$ ago $)$ the cluster was a relatively small $(\sim 10,000$ stars $)$ globular cluster, but got "open cluster" shape due to the dynamical evaporation of the majority of its stars.

\section{Acknowledgements}

This work was began while the first author (S.N.) was an undergraduate student at the Ben-Gurion University. S.N. thanks the Head of Astronomical Programs of the Ilan Ramon Physics Center, Netzach Farbiash, and the Scientific Director of the Ilan Ramon Physics Center, Moshe Schechter, for making possible observations with Meade LX200R telescope. We thank Amir Bernat, Michael Gedalin, and Nimrod Nissim for useful discussions in relation to this work and both anonymous referees for numerous comments that improved the presentation of the paper. Atlas Image obtained as part of the Two Micron All Sky Survey, a joint project of the University of Massachusetts and the Infrared Processing and Analysis Center/California Institute of Technology, funded by the National Aeronautics and Space Administration and the National Science Foundation. This work was sponsored in part by the Israel Science Foundation, the Israeli Ministry of Immigrant Absorption in the framework of the program "KAMEA," and the Binational US-Israel Science Foundation.

\section{REFERENCES}

[1] G. Meynet, J.-C. Mermilliod and A. Maeder, "New Dating of Galactic Open Clusters," Astronomy and Astrophysics Supplement Series, Vol. 98, No. 3, 1993, pp. 477504.

[2] S. Randich, F. Primas, L. Pasquini, P. Sestito and R. Pallavicini, "Tracing Mixing in Stars: New Beryllium Observations of the Open Clusters NGC 2516, Hyades, and M67," Astronomy \& Astrophysics, Vol. 469, No. 1, 2007, pp. 163-172. doi:10.1051/0004-6361:20066218

[3] A. Sarajedini, A. Dotter and A. Kirkpatrick, "Deep 2MASS Photometry of M67," The Astrophysical Journal, Vol. 698, No. 2, 2009, pp. 1872-1878. doi:10.1088/0004-637X/698/2/1872

[4] X. Fan, D. Burstein, J.-S. Chen, J. Zhu, et al., "Deep WideField Spectrophotometry of the Open Cluster M67," The Astrophysical Journal, Vol. 112, No. 2, 1996, pp. 628648. doi:10.1086/118039

[5] D. A. VandenBerg and P. B. Stetson, "On the Old Open Clusters M67 and NGC 188," Publications of the Astronomical Society of Japan, Vol. 116, No. 825, 2004, pp. 997-1011. doi:10.1086/426340

[6] V. Laugalys, A. Kazlauskas, R. P. Boyle, F. J. Vrba, P. A. Davis and V. Straižys, "CCD Photometry of the M67 Cluster in the Vilnius System. II. New Photometry of High Accuracy," Baltic Astron., Vol. 13, 2004, pp. 1-33.

[7] T. M. Girard, W. M. Grundy, C. E. Lopez and W. F. van Altena, "Relative Proper Motions and the Stellar Velocity Dispersion of the Open Cluster M67," Astronomical Journal, Vol. 98, No. 1, 1989, pp. 227-243. doi:10.1086/115139

[8] E. K. Kharadze, R. A. Bartaya, O. B. Dluzhnevskaya, A. E. Piskunov and E. D. Pavlovskaya, "Population of the Galactic Disc in the Solar Neighbourhood. I-Parameters of Spatial Distribution for Stellar Groups of A-K Spectral and III-V Luminosity Classes," Astrophysics and Space Science, Vol. 151, No. 2, 1989, pp. 319-334. doi:10.1007/BF00648388

[9] J. R. Hurley, O. R. Pols, S. J. Aarseth and C. A. Tout, "A Complete N-body Model of the Old Open Cluster M67," Monthly Notices of the Royal Astronomical Society, Vol. 363 , No. 1, 2005, pp. 293-314. doi:10.1111/j.1365-2966.2005.09448.x

[10] C. Bonatto and E. Bica, "Mass Segregation in M67 with 2MASS," Astronomy \& Astrophysics, Vol. 405, No. 2, 2003, pp. 525-530. doi:10.1051/0004-6361:20030205

[11] J. R. A. Davenport and E. L. Sandquist, "Death of a Cluster: The Destruction of M67 as Seen by the Sloan Digital Sky Survey," The Astrophysical Journal, Vol. 711, No. 2, 2010, pp. 559-572. doi: $10.1088 / 0004-637 \mathrm{X} / 711 / 2 / 559$

[12] D. E. McLaughlin, J. Anderson, G. Meylan, K. Gebhardt, C. Pryor, D. Minniti and S. Phinney, "Hubble Space Telescope Proper Motions and Stellar Dynamics in the Core of the Globular Cluster 47 Tucanae," The Astrophysical Journal Supplement Series, Vol. 166, No. 1, 2006, pp. 249-297. doi:10.1086/505692

[13] G. Q. Liu, L. Deng, M. Chavez, E. Bertone, A. H. Davo 
and M. D. Mata-Chavez, "A Spectroscopic Study of the Blue Stragglers in M67," Monthly Notices of the Royal Astronomical Society, Vol. 390, No. 2, 2008, pp. 665674.

[14] J. S. Kalirai, G. G. Fahlman, H. B. Richer and P. Ventura, "The CFHT Open Star Cluster Survey. IV. Two Rich, Young Open Star Clusters: NGC 2168 (M35) and NGC 2323 (M50)," The Astrophysical Journal, Vol. 126, No. 2, 2003, pp. 1402-1414. doi:10.1086/377320

[15] S. Rosseland, "Scattering of Stars in Clusters," Zeitschrift fur Astrophysik, Vol. 4, No. 1, 1932, pp. 235-254.

[16] L. Spitzer, "Distribution of Galactic Clusters," The Astrophysical Journal, Vol. 127, No. 1, 1958, pp. 17-27. doi: $10.1086 / 146435$

[17] L. Spitzer and R. Harm, "Evaporation of Stars from Isolated Clusters," The Astrophysical Journal, Vol. 127, No. 1, 1958, pp. 544-550. doi:10.1086/146486

[18] E. Terlevich, "Evolution of N-body Open Clusters," Monthly Notices of the Royal Astronomical Society, Vol. 224, No. 1, 1987, pp. 193-225.

[19] V. A. Ambartsumyan, "Stability of Stellar Clusters," Uchenye Zapiski LGU, Vol. 22, No. 1, 1938, pp. 19-29.

[20] L. Spitzer, "The Stability of Isolated Clusters," Monthly Notices of the Royal Astronomical Society, Vol. 100, No. 1, 1940, pp. 396-413.

[21] T. A. Agekyan, "The Velocity Distribution Function and the Rate of Dissipation in Systems of Gravitating Bodies," Soviet Astronomy, Vol. 3, No. 1, 1959, pp. 280-290.

[22] S. Chandrasekhar, "Principles of Stellar Dynamics," Dover, New York, 1960.

[23] L. Spitzer and M. H. Hart, "Random Gravitational Encounters and the Evolution of Spherical Systems. I. Method," The Astrophysical Journal, Vol. 164, No. 1, 1971, pp. 399-409. doi:10.1086/150855

[24] R. de Grijs, "The Long-Term Survival Chances of Young Massive Star Clusters," Astrophysics and Space Science, Vol. 324, No. 2-4, 2009, pp. 283-291. doi:10.1007/s10509-009-0100-0

[25] J. Schneider, P. Amaro-Seoane and R. Spurzem, "HigherOrder Moment Models of Dense Stellar Systems: Applications to the Modelling of the Stellar Velocity Distribution Function," Monthly Notices of the Royal Astronomi- cal Society, Vol. 410, No. 1, 2011, pp. 432-454 doi:10.1111/j.1365-2966.2010.17454.x

[26] I. King, "The Escape of Stars from Clusters 11. A Simple Theory of the Evolution of an Isolated Cluster," The Astrophysical Journal, Vol. 63, No. 1258, 1958, pp. 114117. doi:10.1086/107702

[27] S. von Hoerner, "Die Auflsungszeit Offener Sternhaufen," Zeitschrift fur Astrophysik, Vol. 44, No. 1, 1958, pp. 221-242.

[28] L. P. Ossipkov, "On the Jubilee of Academician V. A. Ambartsumyan Statistical Mechanics of Stellar Systems: From Ambartsumyan Onward," Astrophysics, Vol. 51, No. 4, 2008, pp. 428-442. doi:10.1007/s10511-008-9032-6

[29] A. F. Alexandrov, L. S. Bogdankevich and A. A. Rukhadze, "Principles of Plasma Electrodynamics," Springer, Berlin, 1984. doi:10.1007/978-3-642-69247-5

[30] G. G. Kuzmin, "The Effect of Star Encounters and the Evolution of Star Clusters," Publications of Tartu Observatory, Vol. 33, No. 2, 1957, pp. 75-102.

[31] M. N. Rosenbluth, W. M. MacDonald and D. L. Judd, "Fokker-Planck Equation for an Inverse-Square Force," Physical Review, Vol. 107, No. 1, 1957, pp. 1-6. doi:10.1103/PhysRev.107.1

[32] D. C. Montgomery and D. A. Tidman, "Plasma Kinetic Theory," McGraw-Hill, New York, 1964.

[33] B. A. Trubnikov, "Particle Interactions in a Fully Ionized Plasma," In: M. A. Leontovich, Ed., Reviews of Plasma Physics, Consultants Bureau, New York, 1965, pp. 105140.

[34] E. Griv, "Numerical Integration of the Landau Kinetic Equation," In: G. Byrd, Eds., "Order and Chaos in Stellar and Planetary Systems," ASP, San Francisco, 2004, pp. 352-356.

[35] W. M. MacDonald, M. N. Rosenbluth and W. Chuck, "Relaxation of a System of Particles with Coulomb Interactions," Physical Review, Vol. 107, No. 2, 1957, pp. 350-353. doi:10.1103/PhysRev.107.350

[36] J. Killeen and A. H. Futch, "Numerical Solution of the Fokker-Planck Equations for a Hydrogen Plasma Formed by Neutral Injection," Journal of Computational Physics, Vol. 2, No. 3, 1968, pp. 236-254. 


\section{Appendix}

\section{Relaxation of a System of Stars: Numerical Solution of the Fokker-Planck Equation}

Let us consider a system of stars interacting through inverse-square-law Coulombian (Newtonian) forces. As is known, the evolution of an isotropic distribution of like particles (stars) through small-angle Coulomb-like collisions is described by the Fokker-Planck equation for an inverse-square force:

$$
\begin{aligned}
\frac{\partial f}{\partial t} & =2 \pi G^{2} m^{2} \ln \Lambda\left\{\frac{2}{3} \frac{\partial^{2} f}{\partial v^{2}}\left[\int_{0}^{\infty} \mathrm{d} u f u+\frac{1}{v^{3}} \int_{0}^{v} \mathrm{~d} u f u^{4}\right]\right. \\
& +\frac{4}{3 v} \frac{\partial f}{\partial v}\left[\int_{0}^{\infty} \mathrm{d} u f u-\int_{0}^{v} \mathrm{~d} u f u\left(1-\frac{u}{v}\right)^{2}\left(1+\frac{u}{2 v}\right)\right] \\
& \left.+2 f^{2}\right\}
\end{aligned}
$$

where $f(v, t)$ is the distribution function of stars, which is defined by the condition that

$$
n=\int_{0}^{\infty} \mathrm{d} v f v^{2}
$$

is the number density of stars. In this approximation the distribution function depends only on $v$, the magnitude of the velocity, and $t$, the time. Equation (10) has been derived by L. D. Landau (e.g. Ref. [29]) and then fully independent in Ref. [30] for gravitational systems and in Refs. [31,32] for an ionized gas. See Refs. [33,34] for a discussion. It can be shown that, in the absence of sources and losses, the number of particles (stars), as well as total momentum and energy, are conserved within the formalism of the Fokker-Planck equation. The solution of Equation (10), under the same conditions, for a steady state $(\partial f / \partial t=0)$ is the Maxwellian distribution,

$$
f(v) \propto \exp \left(-\frac{v^{2}}{2\left\langle v^{2}\right\rangle}\right) .
$$

Following Refs. [35,36], Equation (10) may be put into dimensionless form

$$
\frac{\partial f}{\partial \tau}=A \frac{\partial^{2} f}{\partial x^{2}}+B \frac{\partial f}{\partial x}+C f,
$$

where $x=v / v_{0}, v_{0}$ is a constant and is a characteristic velocity, $\tau$ is the dimensionless time, and quantities $A$, $B$, and $C$ are defined in Ref. [36]. We integrate this equation directly using the difference equation given in Ref. [36] (see also Ref. [35]).

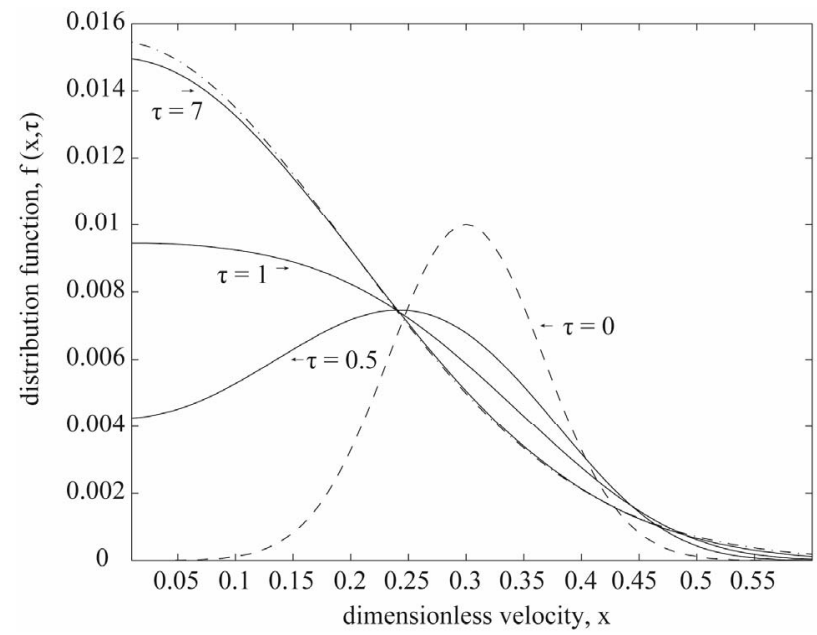

Figure 7. The relaxation of a system of like stars to the equilibrium Maxwellian distribution. The dashed line shows an initial distribution and the dash-dot line shows a Maxwellian of the same density and energy. The time is normalized so that $\tau=1$ corresponds to a ChandrasekharSpitzer relaxation time $t_{\text {chand }}$ (Equation (9)).

We agree with the second referee of this paper that Equation (11) "is neither matching an actual multi-component system nor a spatially inhomogeneous stellar system." This equation can serve only as a convenient starting point for more realistic computations.

We solve Equation (11) numerically, subject to the initial condition that at time $\tau=0$, the initial distribution is $f(x, 0) \propto \exp \left\{-[(x-0.3) / 0.3]^{2}\right\}$. The resulting solution for $f(x, \tau)$ is plotted in Figure 7 for various values of time $\tau$. The final Maxwellian distribution function at $\tau \rightarrow \infty$ is also shown. In fair agreement with the simple Chandrasekhar-Spitzer theory [22, 23], at $\tau \approx 1$ the distribution function of stars becomes Maxwellian-like. As calculations show, near $x \approx 0.25$ and $x \approx 0.45$ the distribution function departs slightly from the equilibrium Maxwellian. However, at $\tau=1$ the lower energy portion of the spectrum is somewhat underpopulated, while the higher energy part is overpopulated. Thus, the higher- and lower-energy parts of the distribution are filled in at a much later time (at times $\tau \gtrsim 10$, Figure 7) as one may expect from the Chandrasekhar-Spitzer theory. We conclude that in quantitative terms the collisional relaxation of the high- and low-energy parts of the distribution is only poorly described by the Chandrasekhar-Spitzer simple "molecularkinetic" theory. 